\title{
Topologies of the Onboard Networks for Small Spacecraft
}

\author{
V.Kh. Khanov, T.V. Borodina \\ Laboratory of Electron Space Device Engineering, Siberian State Aerospace University named \\ after academician M.F. Reshetnev, Krasnoyarsk, Russia
}

Keywords: small spacecraft, board network, SpaceWire.

\begin{abstract}
At present, the network way of spacecraft onboard equipment interaction is becoming widespread. This paper discusses variants of the onboard network topologies for small spacecraft. Examples of the options considered are presented.
\end{abstract}

\section{Introduction}

Network architecture for board equipment interfacing has been widely used in the design of advanced spacecraft lately. It creates a data transmission infrastructure that offers to easily reserve basic infrastructure and network components, to have several alternative ways of data, to scale or modify the network according to the equipment existing on board the spacecraft. The basis for the creation of network architectures is specially designed for space applications network technology SpaceWire [1]. For small spacecraft, the topic is also relevant, because in many cases small spacecraft play the role of a laboratory for developing new technologies, including SpaceWire, prior to their introduction to the full-size spacecraft.

\section{Discussed problems}

SpaceWire allows building a sufficiently extensive network based on the interaction of several routing switches. For small spacecraft onboard network complexity is determined by the natural limitations of functionality and complexity of the spacecraft, due primarily to its small size. On the other hand the choice of topology is influenced by the necessity to ensure a certain level of reliability of the on-board equipment, united in network. Small spacecraft usually fly in low orbits, are not intended for long service life, often have a small financial budget. Therefore, the technical solutions aimed at ensuring the reliability are often significantly simplified. However, at the level of the network topology, there are simple solutions to ensure the reliability of on-board equipment information exchange. And these solutions fully satisfy the resource limitations of small spacecraft. Thus, the paper discusses SpaceWire topologies for small spacecraft, determined mainly by the necessity to ensure the reliability of data transmission.

\section{Variations of onboard network topology}

\section{Network for modular construction of small spacecraft}

A network SpaceWire is made up of a number of links, nodes and routing switches. Nodes of the network are on-board equipment, such as an on-board computer, a command-measuring system, a data acquisition system, various payload equipment, etc. An example of a network, consisting of multiple nodes and routing switches, is shown in Fig. 1. A central routing switch is connected to several peripherals, which in their turn have a connection with the group of nodes.

Such a network topology is consistent with the principle of modular construction of small and micro satellites. They are characterized by scalability of a basic module size UNIT1 designs to a higher dimension UNIT2, UNIT3 etc. The scaling of spacecraft constructive expands network. Not only a routing switch, but also other devices that provide a functional stand-alone module, for example, the computing device and/or the bridge interface integrating other interfaces required for operation of the module into the SpaceWire can increase the versatility of this approach as an infrastructural element of scalability. 


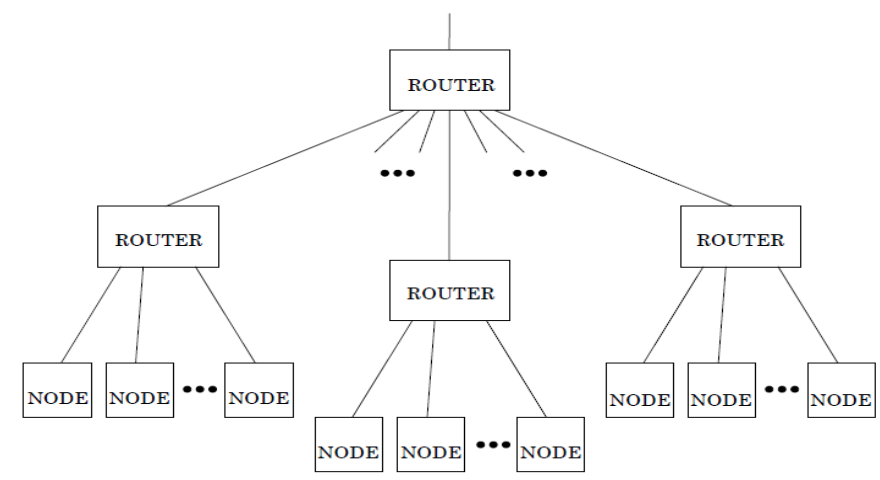

Fig.1: Example of network SpaceWire [1]

However, the network presented in fig. 1 is possible only for small spacecraft weighing more than $100 \mathrm{~kg}$. For most spacecraft grouped in a segment from $1 \mathrm{~kg}$ to $50 \mathrm{~kg}$, it is functionally redundant. The network must be much easier for spacecraft having no high functionality and, consequently, a large number of nodes.

\section{Network providing duplication of onboard equipment}

Most small spacecrafts, having a small number of nodes may be of a simple star type network topology. In this case, operability of the network is vulnerable to failures of routing switches and nodes. Therefore the necessity of reliability forces us to use a double star topology (fig.2).

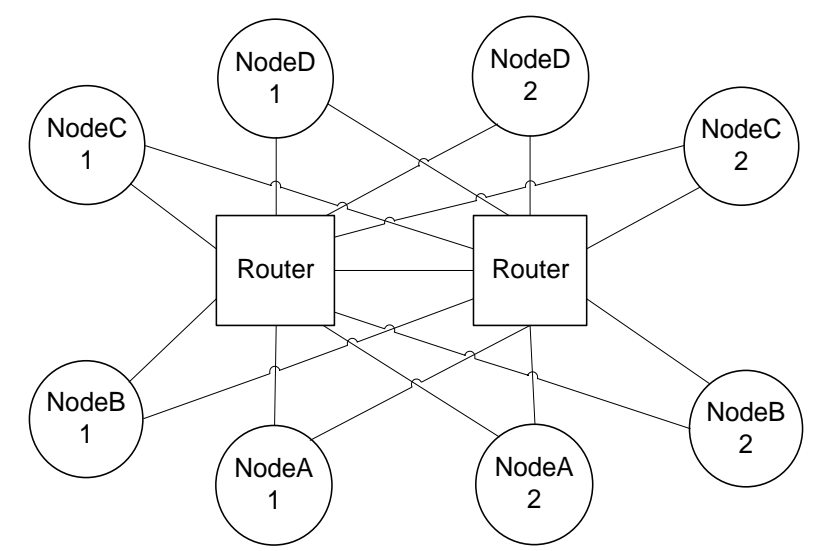

Fig. 2: Network providing duplication of nodes

The presented network involves two routing switches, one of which is active and the other is in cold standby. A set of nodes is connected to each switch. Furthermore, each node of one set is connected to the switch of another set. In real time one set is in the active mode, the other is in cold standby. When a node from one set fails, the analogous node from the other set automatically turns on, and thus, the full structure of the network is saved. When a routing switch fails the operability of network architecture is also preserved. Thus, this topology provides redundant nodes in the onboard network of small spacecraft.

\section{Simple network with redundant links}

In many cases, redundancy of nodes cannot be performed because of the small size, for example, of ultra low spacecrafts, and the network is transformed to a simple star topology. In this case, the refusal of a routing switch completely destroys the network architecture of the spacecraft. However, one can perform a redundancy using the direct links between the nodes and then in case of failure of a routing switch the network operability is saved. Such a network is shown in fig.3.

Each node has its own peripheral routing switch which is linked with a central routing switch. The other two links of a peripheral switch are used for direct connection to the two nearest nodes. A set of direct links between the nodes form a ring. If the central routing switch fails, the data is transferred to the ring. Thus, this topology provides redundant links in the on-board network of small spacecraft. 


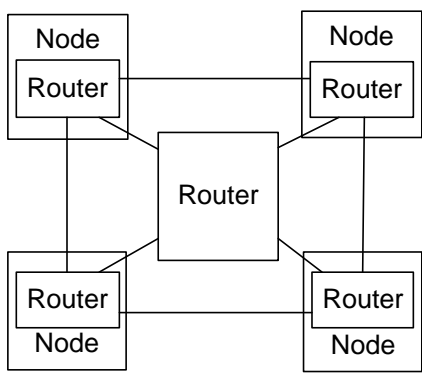

Fig.3: Network with redundant links

\section{Approaches to the implementation of on-board network}

A natural way to implement the network at the physical level is the use of cables. For a complex network (fig. 1) it is the only possible solution. However, it is known that on-board cable network makes a significant contribution to the mass of the spacecraft. The more complex is the network the greater is the mass of the cable network. For many simple small spacecraft, having a small number of nodes, the solution may be a packet (stack) method of linking the network nodes, like the PC 104 specification [2].

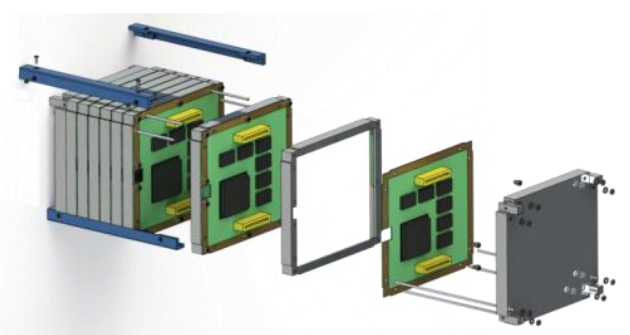

Fig.4: Example of stack design

In this case, each network node is a PCB-device. Communications between devices are provided by separable electrical connectors. In general, the whole structure forms a single radio-electronic unit. If, as shown in Figure 4, each board has two connectors, one is used to supply the network of nodes, and another - for SpaceWire links.

To minimize the length of SpaceWire-links the routing switch must be located in the middle of the stack of network devices. But it does not help to avoid the backhaul chains in contact pairs of releasable connectors providing a connection from node $\mathrm{A}$ to node $\mathrm{B}$, arranged far away from each other. However, the reliability and quality of modern releasable connectors (for example hyperbolic connectors) are high, so taking into account other design solutions this fact can be neglected. Besides it is possible to reduce the speed of SpaceWire, for example, up to $100 \mathrm{Mbit} / \mathrm{s}$. This rate for small spacecraft is more than sufficient.

Note that this method of implementation of the network is natural for many ultra small satellites of the CubeSat, often having a stack structure. For full-size and small spacecraft, this solution is more important in the implementation of the network within a single unit, such as control and data handling device.

\section{Examples of a board network}

Here are a few examples of board network developments for different variants of small spacecraft or its control and data handling device.

The basic infrastructure of on-board network. Development is an example of a network that supports modular design of small spacecraft (fig.5). Basic network infrastructure provides a universal way to build network. It includes the following subsystems: a routing switch, a processor module with a routing switch of a system on a chip (SoC) type and an interface bridge. The development is made on flash FPGA. The SoC-processor is open IP-core Leon3. The maximum 
speed of SpaceWire is $100 \mathrm{Mbit} / \mathrm{s}$. The final stage of development is space execution; the processor unit operates on a small spacecraft TabletSat-Avrora [3].

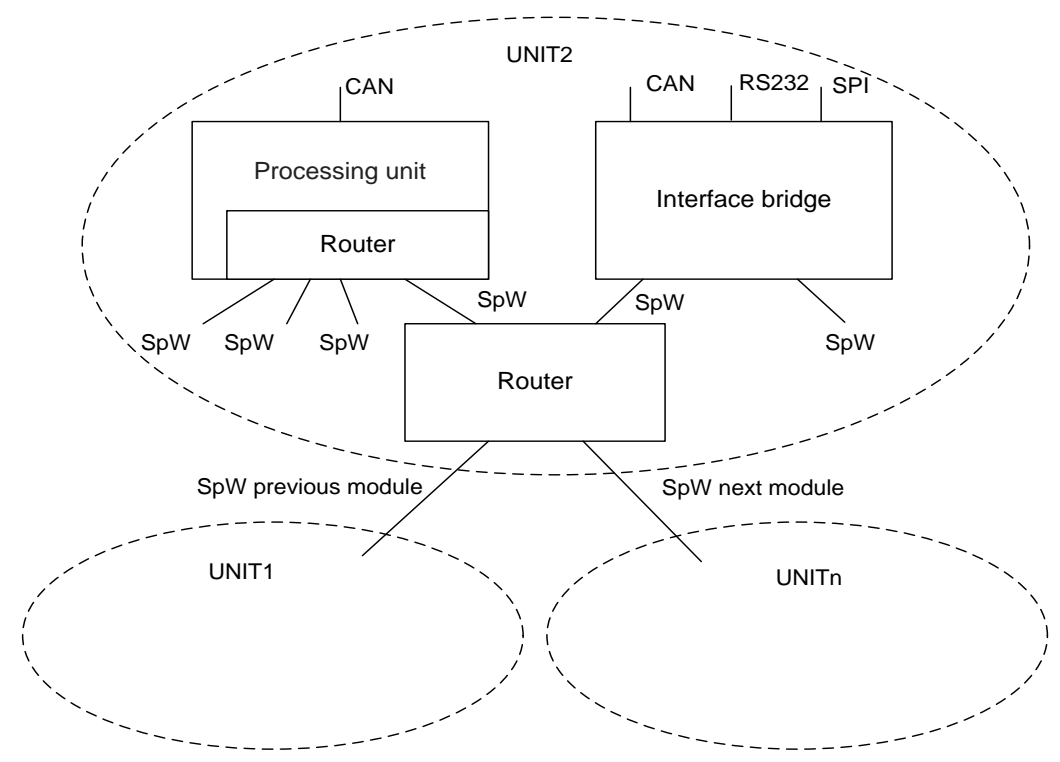

Fig. 5: The basic infrastructure of on-board network

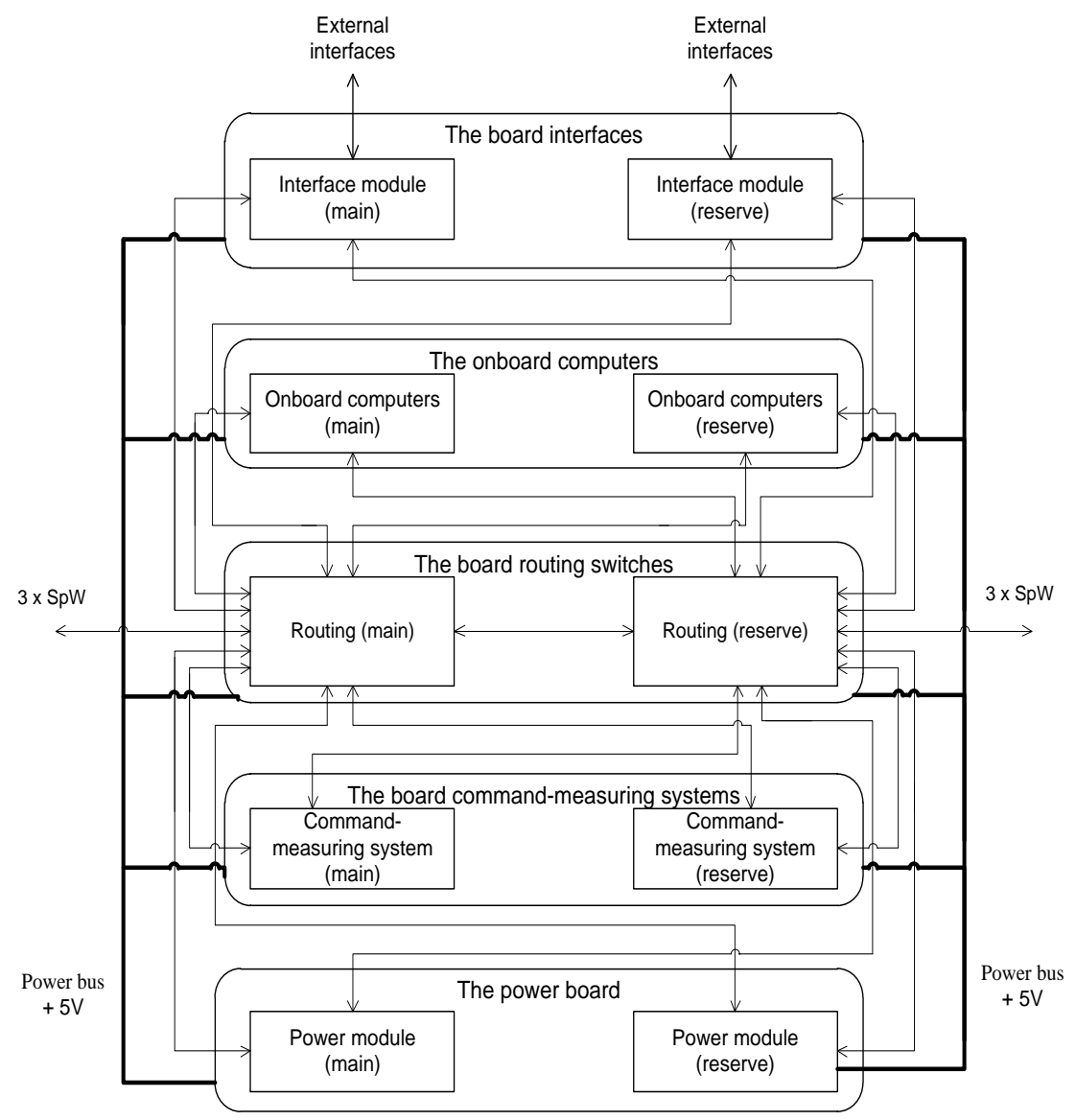

Fig. 6: Network device of control and data handling

Network device of control and data handling ( $\mathbf{C D H})$. The development is an example of a stack design of small spacecraft subsystem consisting of a network. The network topology is a double star. $\mathrm{CDH}$ has two half-sets: the main and the reserve ones (fig.6). It consists of five boards. On each card there are two devices: the main one and a reserve one. These devices are: interface modules, on-board computers, routing switches, command-measuring systems and power modules. All devices are connected to the network routers SpaceWire. A routing switch of each half-set has 9 internal and 3 external ports. Zero switch port has a hardware RMAP-controller for configuration of 
a routing switch. On each board there are three board high-density connectors: one in the center and it is to supply power for devices, the other two on the edges of boards and they link SpaceWire of each half-set. The main element base is flash FPGA. Maximum speed SpaceWire is $100 \mathrm{Mbit} / \mathrm{s}$. Onboard computer is SoC, it has a processor Leon3. The final stage of development is an experimental model.

Network of ultra-small spacecraft. The development is an example of a packet network design of ultra-small spacecraft of CubeSat type. The network topology is a star plus a ring (fig.7). Network nodes are the $\mathrm{CDH}$, the command-measuring system, the orientation system and the two subsystems of payload. Each node has its own router. $\mathrm{CDH}$ is a SoC that integrates the CPU core Leon3, central routing switch and data acquisition subsystem (DAS). The element base development is based on flash FPGA. The maximum speed of SpaceWire is $100 \mathrm{Mbit} / \mathrm{s}$. The current stage of development is conceptual design of ultra-small spacecraft SibCube [4].

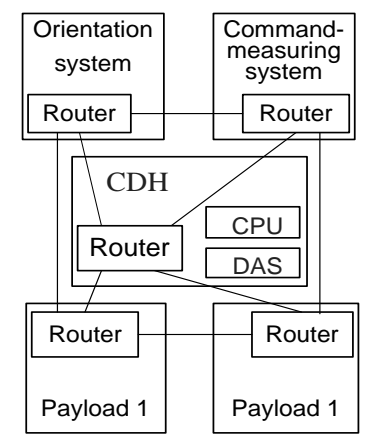

Fig.7: Network of ultra-small spacecraft

\section{Conclusions}

Features of small spacecraft cause restrictions of onboard network complexity. However, the topology can increase the reliability of the network aimed at both the backup node «a double star», and the redundancy links «a star plus a ring».

When designing small spacecraft a network can be implemented both as a distributed collection of nodes linked with cables and in concentrated form as the stack structure integrating PCB-nodes via detachable connectors.

The proposed technical solutions were tested during the creation of several developments of a board network. The results of experimental studies have confirmed the realizability and prospects of the proposed technical solutions.

\section{Acknowledgements}

The research was done with the support of the Ministry of Education and Science of the Russian Federation in accordance with the agreement № 14.574.21.0041, unique identifier RFMEFI57414X0041.

\section{References}

[1] ECSSE-ST-50-12C SpaceWire - Links, nodes, routers and networks. - European Cooperation for Space Standardization (ECSS), 2008 - 129p.

[2] SPECIFICATIONS - PC/104, available at: http://www.pc104.org/ pc104_specs.php.

[3] SPUTNIX has launched the first Russian private Earth remote sensing satellite, available at: http://www.sputnix.ru/en/mediainfo/item/356-sputnix-has-launched-the-first-russian-privateearth-remote-sensing-satellite.

[4] Project SibCube, available at: http://sibcube.sibsau.ru. 